\title{
Cultura Innovadora en Empresas Contratistas de las Minas de Explotación de Carbón en Colombia
}

\author{
Tatiana C. Aroca(1), Vanessa P. Pertuz ${ }^{(2)}$, Adith B. Pérez ${ }^{(2)}$ y Jhon J. Marquez ${ }^{(1)}$ \\ (1) Universidad de Santander. Facultad de ingenierías. Programa de ingeniería Industrial. Valledupar, \\ Colombia. (e-mail: val13151060@mail.udes.edu.co; jho.marquez@mail.udes.edu.co \\ (2) Universidad de Santander. Facultad de ingenierías. Grupo de Investigación Nuevas Tecnologías-UDES. \\ Valledupar, Colombia. (e-mail: van.pertuz@mail.udes.edu.co adi.perez@mail.udes.edu.co)
}

Recibido Feb. 20, 2018; Aceptado Abr. 19, 2018; Versión final May. 21, 2018, Publicado Oct. 2018

\begin{abstract}
Resumen
El artículo analiza los rasgos y los tipos de cultura innovadora en contratistas de empresas mineras del Departamento del Cesar en Colombia. La investigación es cuantitativa y descriptiva. Los resultados indican que el rasgo predominante en las características de la organización dominante, es la concepción de la empresa como una familia extensa. Frente a la gestión de personal, predomina el fomento del trabajo en equipo, el consenso y la participación. Asimismo, el rasgo predominante del vínculo organizacional, es la concepción de la empresa como un espacio caracterizado por la lealtad, el compromiso y la confianza mutua. Por su parte, en el rasgo de criterios de éxito, las empresas promueven el desarrollo de los recursos humanos.
\end{abstract}

Palabras clave: cultura innovadora; cultura organizacional; empresas mineras; rasgos de la cultura innovadora; tipos de cultura innovadora

\section{Innovative Culture in Companies Contractors of the Carbon Exploitation Mines in Colombia}

\begin{abstract}
The features and types of innovative culture in contractors of mining companies at Cesar Department, in Colombia is studied and analyzed. The research is of quantitative and descriptive type. The results indicate that the predominant feature in the characteristics of the dominant organization is the conception of the company as an extended family. With respect to the management of personnel, the promotion of teamwork, consensus and participation predominates among companies. Also, the predominant feature of the organizational link, is the conception of the company as a space characterized by loyalty, commitment and mutual trust. On the other hand, in the feature of success criteria, companies promote the development of human resources.
\end{abstract}

Keywords: innovative culture; organizational culture; mining companies; features of innovative culture; innovative culture types 


\section{INTRODUCCIÓN}

El contexto competitivo en el que se desarrollan las empresas, exige una reconfiguración de los procesos organizacionales basados en el cambio. En este sentido, el concepto de innovación es fundamental en la dinámica empresarial actual, por lo que resulta importante analizar la cultura de la organización como catalizador de procesos innovadores efectivos. Lo anterior, considerando que la innovación implica el desarrollo y la consolidación de un conjunto de habilidades, competencias y destrezas de los colaboradores inmersos en los procesos, que responden a las necesidades de un mercado específico. En este orden de ideas, la cultura de la organización, definida como: la forma de interacción de los individuos de una organización para el logro de los objetivos (Ayala, 2014) o como el conjunto de valores, normas y comportamientos de los miembros de una organización (Bravo y Herrera, 2009); es reconocido en la literatura como un elemento que genera condiciones organizacionales para innovar.

A manera de contexto, la evolución del concepto de innovación incluye diferentes perspectivas, desde Schumpeter (1934) quien la concibe en un sentido general, abarcando: la introducción en el mercado de un nuevo bien, un nuevo método de producción o comercialización de productos, la apertura de un nuevo mercado en el país, la conquista de una nueva fuente de materias primas y la implantación de una nueva estructura. Posteriormente, el Manual de Oslo define la innovación como la implementación de un producto o proceso, nuevo o significativamente mejorado, un nuevo método de comercialización, o un nuevo método organizacional en las prácticas de negocio, la organización del lugar de trabajo o las relaciones externas (Manual de Oslo de OECD y Eurostat, 2005). Paralelamente, el Manual de Bogotá, incluye en el concepto de innovación el "esfuerzo tecnológico", correspondiente a toda acción llevada a cabo por una empresa con el objeto de implementar conceptos, ideas y métodos necesarios para adquirir, asimilar e incorporar nuevos conocimientos (RICYT /OAS /CYTED /COLCIENCIAS /OCYT, 2001).

Consecuentemente, las investigaciones que relacionan la cultura con la capacidad de innovación han concluido que elementos de la cultura vinculados a la creación de normas para mejorar la creatividad, están asociados a un alto grado de innovación (Bravo y Herrera, 2009). En el mismo sentido, Naranjo y Calderón (2015) destacan que la cultura organizacional es un determinante clave de la innovación, no obstante, esta puede favorecer o dificultar la innovación en función de cuáles sean los rasgos o valores que fomente y, por lo tanto, los comportamientos que estimule. Así, Rosenbusch et al. (2011) concluyen que, para las pymes es más beneficiosa una orientación estratégica de la innovación, que el hecho de centrarse en el desarrollo de productos innovadores. Así, la capacidad de innovación es una competencia distintiva que permite innovar $y$, consecuentemente, potenciar la competitividad, especialmente en las pequeñas y medianas empresas (Fernández, 2012).

En contraste, la implementación de procesos de innovación en el sector productivo en Colombia, presenta deficiencias significativas. Esta premisa se valida mediante el análisis de los resultados de las Encuesta de Desarrollo e Innovación Tecnológica, en el sector industrial (EDIT) y en el sector servicios (EDITS), realizadas el Colombia, por el Departamento Administrativo Nacional de Estadísticas DANE, con el objeto de caracterizar la dinámica de innovación de las empresas del país (Departamento Administrativo Nacional de Estadísticas, 2015). Las encuestas en referencia categorizan a las empresas en: innovadoras en sentido estricto, innovadoras en sentido amplio, potencialmente innovadoras y no innovadoras. En concordancia con lo anterior, los resultados de la EDIT para el periodo 2013- 2014, con una población de ocho mil ochocientos treinta y cinco (8.835) empresas del sector industrial, clasifican al $0,1 \%$ como innovadoras en sentido estricto; el 19,3\% como innovadoras en sentido amplio, seguido de un $3,8 \%$ consideradas potencialmente innovadoras y el $76,8 \%$ como no innovadoras (Departamento Administrativo Nacional de Estadísticas DANE, 2015). Por otro lado, la Encuesta de Desarrollo e Innovación Tecnológica en el sector servicios (EDITS), para el periodo 2012-2013, consideró una población de cinco mil ochocientos cuarenta y ocho (5.848) empresas. Los resultados indican que el $0,05 \%$ de las empresas, se consideran innovadoras en sentido estricto; seguidos de un $23,84 \%$ clasificadas como innovadoras en sentido amplio; el $4,33 \%$ son potencialmente innovadoras, mientras que el $71,79 \%$ restante se categorizan como no innovadoras (Departamento Administrativo Nacional de Estadísticas DANE, 2014).

En referencia a las encuestas anteriormente enunciadas, González (2013) indica que los resultados obtenidos de las diferentes aplicaciones realizadas en el país desde mediados de los años noventa, demuestran falencias de las empresas colombianas frente a elementos claves para la innovación. Así, las deficiencias de las empresas en materia de innovación, inciden en la competitividad de las mismas, y en su capacidad para generar valor agregado y ventajas competitivas sostenibles en el tiempo. Paralelamente, Arancibia et al. (2015) señalan que, en el contexto chileno, la cultura de la innovación se encuentra en un proceso emergente, debido a que se debe hacer frente a los cambios sociales y ambientales del entorno, a las exigencias de los mercados, a la búsqueda de maximización de la productividad y a la sustentabilidad de una empresa, exigiendo mejoras en las estrategias competitivas. En consecuencia, el objetivo del presente 
artículo es analizar los rasgos y los tipos de cultura innovadora en las empresas contratistas de las minas de explotación de carbón del departamento del Cesar, Colombia, para potencializar la innovación y la competitividad sectorial, en acuerdo con la evidencia disponible en la literatura.

\section{RASGOS Y TIPO DE CULTURA INNOVADORA: REVISIÓN DE ANTECEDENTES}

En la revisión de literatura se presentan antecedentes asociados al estudio de la cultura innovadora en diferentes contextos: los autores, Arancibia et al. (2015) identifican los factores clave en la cultura de innovación, en el caso de la mediana minería en Chile. Para tal fin se establece un modelo de medición del nivel de la cultura de innovación basado en los postulados de Naranjo et al. (2012) y OECD y Eurostat (2005). Los resultados destacan la importancia del recurso humano como factor clave para estimular la cultura de innovación; así, para elevar la cultura de innovación se requiere un mayor énfasis en innovaciones organizacionales asociadas a la gestión del conocimiento, la vigilancia tecnológica y la inteligencia competitiva. Paralelamente, Tejeiro (2014) realiza la medición de la cultura de innovación, mediante cuatro estudios de caso, con el objeto de elaborar una herramienta para la medición de la cultura corporativa que impulse la innovación, en la misma línea del trabajo de Naranjo et al. (2012).

De otro lado, Souto (2015) analiza la gestión de una cultura de innovación basada en las personas; con el objeto de proponer una herramienta de gestión de cultura de innovación basada en las personas, desde una perspectiva del trabajo en equipo. Lo anterior, debido a que la innovación se presenta solo mediante la aglutinación de los esfuerzos de toda la empresa; de esta manera, la cultura innovadora es fundamental para generar capacidad de innovación en las empresas. Por su parte, Guzmán (2015) estudian la innovación empresarial y la cultura organizacional como elementos complementarios y necesarios entre sí, para afrontar el futuro; encontrando que una cultura empresarial sólida, abierta, rigurosa, pero, al mismo tiempo flexible y abierta al cambio es un factor importante para fomentar la innovación empresarial. Adicionalmente, es relevante establecer rutinas organizativas orientadas a favorecer la creatividad y la innovación (Guzmán, 2015).

A nivel Colombia, Pertuz y Perez (2016) proponen un modelo de cultura organizacional innovadora en caficultores del departamento del Cesar, basado en los planteamientos de Naranjo y Calderón (2015), Naranjo et al. (2012) y Ayala (2014), destacando que el desarrollo de una cultura innovadora se concibe como un proceso cíclico, consistente en un conjunto de etapas direccionadas a la generación de conductas y comportamientos para impulsar el desarrollo innovador. De la misma manera, Sanabria et al. (2015) analizan las condiciones para la innovación, cultura organizacional y sostenibilidad de las organizaciones. En este sentido, la empresa se concibe como un producto social diacrónico que responde a los niveles de incremento de adaptación y complejidad, orientada a reducir el riesgo e incertidumbre para transformar el entorno; por lo que, la sostenibilidad de la empresa se refleja en su capacidad interpretativa a partir de su estructura estratégica adaptativa, desarrollada como juego de cooperación y competitividad en sus variantes de precios o creatividad (Sanabria et al., 2015).

De otro modo, Ruiz y Naranjo (2012) analizan la cultura organizacional en Colombia desde la perspectiva de la difusión en revistas científicas indexadas en el periodo comprendido entre 2006 y 2010, concluyendo que cada vez existe más evidencia sobre el papel determinante de la cultura organizacional para lograr mejores niveles de desempeño y competitividad en las empresas, lo cual ha generado un creciente interés por el estudio de la cultura organizacional, sin embargo, en el caso de Colombia no se ha indagado sobre la investigación realizada en el tema. Los autores Ruiz y Naranjo (2012) realizan una revisión de literatura de la cultura organizacional en Colombia, considerando, entre otros, los trabajos de Cameron y Quinn (1999) y Calderón y Naranjo (2007), estableciendo que la literatura de cultura organizacional en Colombia se enfoca en realidades concretas: clúster, empresas innovadoras, empresas exportadoras, sectores específicos como el metalmecánico, y se interesa en analizar cómo debe gestionarse la cultura para articularse adecuadamente con otras variables organizacionales. Por su parte, Calderón y Naranjo (2007) establecen el perfil cultural de las empresas innovadoras, desde un estudio de caso en seis empresas metalmecánicas clasificadas como innovadoras, mediante la aplicación del test de Bridges. En este sentido, el perfil corresponde a empresas abiertas al entorno, sensibles a las señales del mercado y del entorno; con flexibilidad a la programación de actividades e innovadoras incrementales en la mayoría de los casos.

Así, las investigaciones de Naranjo et al. (2012) analizan la cultura innovadora desde el modelo de valores en competencia (MVC) de Cameron y Quinn (1999), que clasifica la cultura en cuatro tipologías: de clan, adhocrática, jerárquica y racional. De acuerdo con el modelo propuesto por Cameron y Quinn (1999), una cultura adhocrática valora más la flexibilidad que la estabilidad y tiene orientación externa; promueve valores como la iniciativa, la creatividad y la asunción de riesgos (Naranjo et al., 2012). Por su parte, la cultura de clan da importancia a la flexibilidad y a la orientación interna, enfatizando en el desarrollo humano, el trabajo en equipo, la participación de los trabajadores y el compromiso de todos con la 
organización. La cultura de mercado, enfatiza en lo externo, pero requiere estabilidad y control para mejorar su productividad y competitividad; por lo que potencia la consecución de objetivos ambiciosos. La cultura jerárquica tiene una orientación interna y busca la estabilidad y el control; mediante la eficiencia, el cumplimiento de las normas y la formalización de los procesos y la estabilidad del personal (Naranjo et al., 2012).

La tipología de Cameron y Quinn (1999) es la más extendida y ampliamente aceptada en la literatura (Naranjo y Calderón, 2015). De acuerdo con las conclusiones de los autores en referencia, cada uno de estos tipos de cultura tiene efectos distintos sobre la innovación. Al respecto, la cultura de tipo adhocrática favorece la innovación, mientras que la de tipo jerárquico la afecta negativamente (Naranjo y Calderón, 2015) Consecuentemente, de los cuatro tipos de cultura definidos de acuerdo al modelo de valores en competencia propuesto por Cameron y Quinn (1999) pueden definirse los factores o rasgos organizativos: las características dominantes de la organización, esto es, cómo es la organización en su conjunto; la gestión de personal o recursos humanos, esto es, cuáles son los principios básicos que orientan la gestión de los empleados y cómo es el ambiente de trabajo; el vínculo organizacional, o mecanismo que ayuda a la organización a permanecer unida; y el criterio de éxito, que hace referencia a cómo se define el triunfo en la organización y qué se recompensa y elogia (Naranjo et al., (2012).

Adicionalmente, la investigación de Naranjo et al. (2012) indican que cuando las características dominantes, es decir, la concepción general de cómo es la empresa y el criterio de éxito, es decir, cómo se define el triunfo en la empresa, coinciden con los de la cultura adhocrática, se produce un mejoramiento en los procesos de innovación. Lo anterior, debido a que, según los autores, lo que limita la innovación no es el hecho de que la empresa esté jerarquizada, formalizada y estructurada, sino que, el respeto de esas normas y políticas formales y el cumplimiento de la jerarquía sean el valor cultural más importante que comparten todos los miembros de la empresa (Naranjo et al., 2012). En el mismo sentido, Mohr et al. (2010) señalan que la cultura adhocrática es la mejor base para la capacidad de innovación radical de producto, dado que se caracteriza por los valores de la iniciativa empresarial, la innovación, la adaptabilidad, la propensión por el riesgo, así como una orientación externa.

De otra manera, una gestión de personal de tipo adhocrática impacta positivamente en la innovación Siguaw et al. (2006), mientras que la gestión de personal asociada a las culturas jerárquicas supone un obstáculo para la innovación (Naranjo et al., 2012). Adicionalmente, cuando la gestión de personal pone el énfasis en el trabajo en equipo la innovación es mayor (Naranjo et al., 2012). De otro lado, en el caso de la cultura de clan el rasgo de gestión del personal incluye el trabajo en equipo, factor que la literatura asocia positivamente con la innovación (Naranjo et al., 2012).

En referencia al vínculo organizacional, relacionado con los valores compartidos por los colaboradores, los resultados de Naranjo et al. (2012) señalan que el compromiso con la innovación y el cambio, son valores que generan un efecto positivo al proceso innovador, mientras que, los valores correspondientes a las jerarquías y el respecto a las reglas y políticas formales afectan negativamente la innovación. De este modo, el vínculo organizacional es el rasgo que más claramente se asocia a la innovación, lo que indica que para fomentar la innovación debe analizarse los valores compartidos por los miembros de la organización, asimismo, estimular la asunción de riesgos y la implementación exitosa de productos en el mercado (Naranjo et al., 2012).

Asimismo, López y Robledo (2014) destacan que además de contar con personas creativas en la organización, es necesario gestionar el proceso de creatividad, lo cual implica una cultura con destrezas tales como: la motivación del personal la flexibilidad y personalización de las políticas y esquemas motivacionales a través de las aéreas de talento humano. De otro lado, de acuerdo con Saunila y Ukko (2014) la cultura de liderazgo participativo se relaciona con el ambiente general que apoya y motiva la innovación, así como las prebendas y acciones de los lideres (Saunila et al., 2014), (Saunila y Ukko, 2014) y (López y Robledo, 2014). Por su parte, Mohr et al. (2010) resaltan la importancia del líder en el proceso de innovación. Al respecto, es importante destacar que, pese a la relevancia de la cultura organizacional para estimular la innovación, la investigación empírica en este aspecto ha sido bastante limitada (Naranjo et al., 2012). Los autores en referencia destacan las siguientes características de una cultura innovadora: la creatividad, la libertad/autonomía, la tolerancia al riesgo, el trabajo en equipo, la suficiencia de recursos, la orientación estratégica hacia el cliente, la toma de decisiones, la participación de los trabajadores, el aprendizaje continuo y la flexibilidad. Adicionalmente, resulta importante considerar el aprendizaje para desarrollar procesos de innovación (Rueda y Rodenes, 2010).

\section{METODOLOGÍA}

La investigación se aborda desde el enfoque cuantitativo (Hernandez et al., 2014); de tipo descriptivo (Hernandez et al., 2014). Adicionalmente, el estudio se utiliza una metodología de campo, no experimental y 
transeccional (Hernandez et al., 2014). Las unidades de análisis son la totalidad de empresas contratistas (95) asociadas a las actividades de operación de un grupo minero. La investigación considera un censo poblacional de las empresas que accedieron a contestar el cuestionario y empresas que asistieron a las instalaciones de la mina en el periodo de recolección de la información; obteniendo 26 empresas para el desarrollo de la investigación. Las características de estas empresas se detallan en la Tabla 1.

Tabla 1: Características de las empresas objeto de estudio

\begin{tabular}{|c|c|c|c|c|c|c|}
\hline $\begin{array}{c}\text { Nombre de la } \\
\text { empresa }\end{array}$ & $\begin{array}{c}N^{\circ} \text { de } \\
\text { trabajad } \\
\text { ores } \\
\end{array}$ & $\begin{array}{l}\text { Antigüed } \\
\text { ad }\end{array}$ & $\begin{array}{l}\text { Tipo de } \\
\text { capital }\end{array}$ & Principales clientes & $\begin{array}{l}\text { Información } \\
\text { financiera }\end{array}$ & $\begin{array}{c}\text { Servicios que } \\
\text { provee a la mina } \\
\text { objeto de estudio }\end{array}$ \\
\hline $\begin{array}{l}\text { Dimantec } \\
\text { LTDA }\end{array}$ & 956 & 30 años & $\begin{array}{l}\text { Privada, } \\
\text { nacional }\end{array}$ & $\begin{array}{l}\text { Prodeco. } \\
\text { No disponible } \\
\text { información del resto } \\
\text { de clientes }\end{array}$ & $\begin{array}{c}\$ 37,384,251 \\
\text { Activos totales. } \\
2016 \text { (Unidades } \\
\text { en Miles) }\end{array}$ & $\begin{array}{l}\text { Mantenimiento y } \\
\text { reparación } \\
\text { especializada de } \\
\text { maquinaria y } \\
\text { equipo. }\end{array}$ \\
\hline $\begin{array}{c}\text { Relianz } \\
\text { Mining } \\
\text { Solutions S A } \\
\text { S }\end{array}$ & - & 4 años & $\begin{array}{l}\text { Nacional, } \\
\text { privada }\end{array}$ & $\begin{array}{c}\text { Industrias de minería } \\
\text { subterránea y } \\
\text { minería superficie }\end{array}$ & $\begin{array}{c}\$ 565,005,330 \\
\text { Activos totales. } \\
2016 \text { (Unidades } \\
\text { en Miles) }\end{array}$ & $\begin{array}{c}\text { Proveedor de } \\
\text { productos para } \\
\text { minería (equipos } \\
\text { nuevos, } \\
\text { reconstrucción de } \\
\text { componentes } \\
\text { calidad } \\
\text { CATERPILLAR) }\end{array}$ \\
\hline $\begin{array}{l}\text { CHM Minería } \\
\text { SAS }\end{array}$ & 1,642 & 5 años & $\begin{array}{l}\text { Nacional, } \\
\text { privada }\end{array}$ & $\begin{array}{c}\text { Grupo Prodeco, } \\
\text { Carbones Del } \\
\text { Cerrejon Limited, } \\
\text { South 32, Argos, } \\
\text { Mapesa }\end{array}$ & $\begin{array}{c}\$ 254,238,055 \\
\text { Activos totales. } \\
2016 \text { (Unidades } \\
\text { en Miles) }\end{array}$ & $\begin{array}{c}\text { Dedicada a la } \\
\text { comercialización de } \\
\text { equipos, repuestos, } \\
\text { servicios de } \\
\text { mantenimiento y } \\
\text { asistencia técnica } \\
\text { especializada para } \\
\text { la industria minera, } \\
\text { portuaria y de } \\
\text { construcción }\end{array}$ \\
\hline $\begin{array}{c}\text { Austin } \\
\text { Ingenieros } \\
\text { Colombia S } \\
\text { A S } \\
\end{array}$ & 450 & $\begin{array}{l}36 \text { años } \\
8 \text { años en } \\
\text { Colombia }\end{array}$ & $\begin{array}{l}\text { Internacion } \\
\text { al, privada }\end{array}$ & $\begin{array}{l}\text { Mineros, Contratistas } \\
\text { Mineras Y Fabricantes } \\
\text { De Equipos Originales }\end{array}$ & $\begin{array}{c}\$ 33,673,777 \\
\text { Activos totales. } \\
2016 \text { (Unidades } \\
\text { en Miles) } \\
\end{array}$ & $\begin{array}{l}\text { Fabricantes de } \\
\text { equipos mineros }\end{array}$ \\
\hline $\begin{array}{c}\text { Servicios } \\
\text { Integrales } \\
\text { Confiables } \\
\text { de Ingeniería } \\
\text { SAS }\end{array}$ & 62 & 3 años & $\begin{array}{l}\text { Nacional, } \\
\text { privada }\end{array}$ & $\begin{array}{c}\text { Universidad Popular } \\
\text { del Cesar, Mina } \\
\text { Calenturitas, } \\
\text { Electricaribe, Land } \\
\text { Developers Colombia } \\
\text { SAS }\end{array}$ & 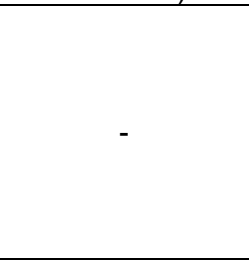 & $\begin{array}{c}\text { Servicios integrales } \\
\text { en la construcción } \\
\text { de redes eléctricas, } \\
\text { servicios de } \\
\text { comercialización y } \\
\text { mantenimiento de } \\
\text { equipos eléctricos }\end{array}$ \\
\hline $\begin{array}{l}\text { C I Prodeco } \\
\text { S A } \\
\text { (Multinaciona } \\
\text { I Glencore) }\end{array}$ & 2547 & 44 años & $\begin{array}{l}\text { Privada, } \\
\text { internacion } \\
\text { al }\end{array}$ & $\begin{array}{c}\text { Mina Calenturitas y la } \\
\text { mina La Jagua en el } \\
\text { Cesar }\end{array}$ & $\begin{array}{c}\$ 3,703,640,565 \\
\text { Activos totales. } \\
2016 \text { (Unidades } \\
\text { en Miles) }\end{array}$ & $\begin{array}{l}\text { Exportación de } \\
\text { carbón térmico y } \\
\text { metalúrgico }\end{array}$ \\
\hline $\begin{array}{l}\text { Rg - Mass } \\
\text { Construccion } \\
\text { es Civiles } \\
\text { S.A.S. }\end{array}$ & - & 5 años & $\begin{array}{l}\text { Nacional, } \\
\text { privada }\end{array}$ & $\begin{array}{c}\text { Prodeco. } \\
\text { No disponible } \\
\text { información del resto } \\
\text { de clientes }\end{array}$ & 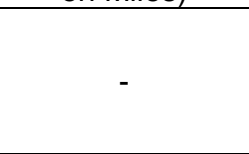 & $\begin{array}{c}\text { Asesoría y } \\
\text { desarrollo del } \\
\text { proyectos y } \\
\text { construcciones }\end{array}$ \\
\hline $\begin{array}{c}\text { Tech } \\
\text { Ingenieria JV } \\
\text { S A S }\end{array}$ & - & 27 años & $\begin{array}{l}\text { Nacional, } \\
\text { privada }\end{array}$ & $\begin{array}{c}\text { Prodeco. } \\
\text { No disponible } \\
\text { información del resto } \\
\text { de clientes }\end{array}$ & $\begin{array}{c}\text { Entre } \\
2.000 .000 .000 \mathrm{y} \\
5.000 .000 .000 \\
\text { COP (Ventas) }\end{array}$ & $\begin{array}{l}\text { Construcción de } \\
\text { carreteras y vías de } \\
\text { ferrocarril. }\end{array}$ \\
\hline $\begin{array}{l}\text { Perforacione } \\
\text { s Caroni } \\
\text { Drilling } \\
\text { Colombia } \\
\text { S.A.S }\end{array}$ & 50 & 7 años & $\begin{array}{l}\text { Nacional, } \\
\text { privada }\end{array}$ & $\begin{array}{c}\text { Coal Corp,Cerrejon, } \\
\text { Carbones del Caribe, } \\
\text { Prodeco, Vale Coal } \\
\text { Colombia, Carbones } \\
\text { de la Jagua, } \\
\text { Cermentos Argos, }\end{array}$ & $\begin{array}{c}\$ 3,434,965 \\
\text { Activos totales. } \\
2016 \text { (Unidades } \\
\text { en Miles) }\end{array}$ & $\begin{array}{l}\text { Líder en } \\
\text { perforaciones } \\
\text { geológicas }\end{array}$ \\
\hline $\begin{array}{l}\text { Chaneme } \\
\text { Comercial } \\
\text { SA }\end{array}$ & 339 & 36 años & $\begin{array}{c}\text { Nacional } \\
\text { privada }\end{array}$ & $\begin{array}{c}\text { Conasfaltos, Holcim, } \\
\text { MHC, Metronics, } \\
\text { Prodeco, SITP, Argos, } \\
\text { AGM Desarrollo, EI } \\
\text { Codor SA, Compass, } \\
\text { Inmunizar, Gisaico }\end{array}$ & $\begin{array}{c}\$ 201,893,622 \\
\text { Activos totales. } \\
2016 \text { (Unidades } \\
\text { en Miles) }\end{array}$ & $\begin{array}{l}\text { Producción de } \\
\text { autopartes }\end{array}$ \\
\hline
\end{tabular}




\begin{tabular}{|c|c|c|c|c|c|c|}
\hline \multicolumn{7}{|c|}{ Tabla 1 (continuación) } \\
\hline $\begin{array}{c}\text { Kal Tire } \\
\text { Mining Tire } \\
\text { Group }\end{array}$ & 163 & $\begin{array}{l}40 \text { años } \\
13 \text { años } \\
\text { en } \\
\text { Colombia }\end{array}$ & $\begin{array}{l}\text { Internacion } \\
\text { al, privada }\end{array}$ & $\begin{array}{l}\text { Empresas de minería } \\
\text { a cielo abierto o en } \\
\text { minas subterráneas, } \\
\text { canteras, puertos o } \\
\text { sitios de construcción }\end{array}$ & $\begin{array}{c}\$ 16,239,560 \\
\text { Activos totales. } \\
2016 \text { (Unidades } \\
\text { en Miles) }\end{array}$ & $\begin{array}{c}\text { Suministro y } \\
\text { administración en } \\
\text { sitio para el } \\
\text { mantenimiento de } \\
\text { neumáticos. } \\
\end{array}$ \\
\hline $\begin{array}{c}\text { Tecno Fuego } \\
\text { S.A.S. }\end{array}$ & 177 & 34 años & $\begin{array}{l}\text { Nacional, } \\
\text { privada }\end{array}$ & $\begin{array}{c}\text { Prodeco. } \\
\text { No disponible } \\
\text { información del resto } \\
\text { de clientes }\end{array}$ & $\begin{array}{c}\$ 24,153,341 \\
\text { Activos totales. } \\
2016 \text { (Unidades } \\
\text { en Miles) }\end{array}$ & $\begin{array}{l}\text { Evaluación, estudio } \\
\text { y protección } \\
\text { especializada de } \\
\text { todo tipo de riesgos } \\
\text { de incendio }\end{array}$ \\
\hline $\begin{array}{l}\text { Servisold } \\
\text { S.A.S }\end{array}$ & 150 & 5 años & $\begin{array}{l}\text { Privada, } \\
\text { nacional }\end{array}$ & Prodeco, PLJ & - & $\begin{array}{l}\text { Mantenimiento, } \\
\text { fabricación, } \\
\text { reparación de } \\
\text { equipos mediante } \\
\text { procesos de } \\
\text { soldadura }\end{array}$ \\
\hline $\begin{array}{c}\text { Incolab } \\
\text { Services } \\
\text { Colombia S } \\
\text { A S }\end{array}$ & 149 & 23 años & $\begin{array}{l}\text { internacion } \\
\text { al, privada }\end{array}$ & $\begin{array}{l}\text { Laboratorio de } \\
\text { Ensayos acreditado } \\
\text { por ONAC en las } \\
\text { áreas acreditadas de } \\
\text { Petróleo, carbón y } \\
\text { productos derivados }\end{array}$ & $\begin{array}{c}\$ 10,852,189 \\
\text { Activos totales. } \\
2016 \text { (Unidades } \\
\text { en Miles) }\end{array}$ & $\begin{array}{l}\text { Control de calidad y } \\
\text { cantidad de } \\
\text { combustibles } \\
\text { sólidos, } \\
\text { especializados en } \\
\text { carbón y coque }\end{array}$ \\
\hline $\begin{array}{l}\text { Fluiconnecto } \\
\text { Servicios } \\
\text { Hidráulicos } \\
\text { S.A.S }\end{array}$ & 43 & $\begin{array}{l}80 \text { años } \\
17 \text { años } \\
\text { en } \\
\text { Colombia }\end{array}$ & $\begin{array}{l}\text { Privada, } \\
\text { internacion } \\
\quad \text { al }\end{array}$ & $\begin{array}{l}\text { Empresas de los } \\
\text { sectores de } \\
\text { construcción y obras } \\
\text { públicas } \\
\text { Minería } \\
\text { Gas y petróleo } \\
\text { Agricultura } \\
\text { Industria y fabricación }\end{array}$ & $\begin{array}{c}\$ 7,452,464 \\
\text { Activos totales. } \\
2016 \text { (Unidades } \\
\text { en Miles) }\end{array}$ & $\begin{array}{c}\text { Mayorista de } \\
\text { mangueras } \\
\text { hidráulicas e } \\
\text { industriales y } \\
\text { acoples metálicos. }\end{array}$ \\
\hline $\begin{array}{c}\text { Grúas } \\
\text { Maniobras Y } \\
\text { Montajes S A } \\
\text { S }\end{array}$ & 68 & 22 años & $\begin{array}{l}\text { Nacional, } \\
\text { privada }\end{array}$ & $\begin{array}{c}\text { Boom Logísticas S.A., } \\
\text { Servicios } \\
\text { Elesctrohidráulicos } \\
\text { Ltda., Astilleros } \\
\text { Unidos S.A. }\end{array}$ & $\begin{array}{c}\$ 23,014,561 \\
\text { Activos totales. } \\
2016 \text { (Unidades } \\
\text { en Miles) }\end{array}$ & $\begin{array}{c}\text { Movimiento de } \\
\text { cargas de gran peso } \\
\text { y volumen }\end{array}$ \\
\hline $\begin{array}{c}\text { Bandas y } \\
\text { Servicios de } \\
\text { la Costa SAS }\end{array}$ & 36 & 9 años & $\begin{array}{l}\text { Nacional, } \\
\text { privada }\end{array}$ & $\begin{array}{c}\text { Puerto Nuevo S.A, } \\
\text { Barranquilla } \\
\text { International Terminal } \\
\text { Company, Malteria } \\
\text { Tropical, AGM } \\
\text { Desarrollos, Prodeco, } \\
\text { Metalplast, Schrander } \\
\text { Camargo, Bedeschi, } \\
\text { Argos, Trituradora } \\
\text { AGM }\end{array}$ & $\begin{array}{c}\$ 6,136,352 \\
\text { Activos totales. } \\
2016 \text { (Unidades } \\
\text { en Miles) }\end{array}$ & $\begin{array}{l}\text { Comercialización, } \\
\text { instalación y } \\
\text { mantenimiento de } \\
\text { bandas } \\
\text { transportadoras }\end{array}$ \\
\hline $\begin{array}{l}\text { Consorcio } \\
\text { Dia S.A. }\end{array}$ & 30 & 4 años & $\begin{array}{l}\text { Privada, } \\
\text { nacional }\end{array}$ & $\begin{array}{l}\text { Prodeco, Cerrejón, } \\
\text { Avanade, Operadores } \\
\text { Logístico del Caribe, } \\
\text { Chevron, Drummond } \\
\text { Limited, Biomax, } \\
\text { Terpel, Impala }\end{array}$ & $\begin{array}{c}\$ 5,695,429 \\
\text { Activos totales. } \\
2016 \text { (Unidades } \\
\text { en Miles) }\end{array}$ & $\begin{array}{l}\text { Prestación de } \\
\text { servicios logísticos } \\
\text { de transporte de } \\
\text { carga liquida y carga } \\
\text { seca }\end{array}$ \\
\hline $\begin{array}{l}\text { Transporte } \\
\text { Sensación } \\
\text { Ltda }\end{array}$ & - & 24 años & $\begin{array}{l}\text { Nacional, } \\
\text { privada }\end{array}$ & $\begin{array}{l}\text { Servicio de transporte } \\
\text { de personal para } \\
\text { diferentes empresas }\end{array}$ & $\begin{array}{c}\text { Entre } \\
5.000 .000 .000 \mathrm{y} \\
10.000 .000 .000 \\
\text { COP (Ventas) }\end{array}$ & $\begin{array}{l}\text { Servicio especial y } \\
\text { de turismo de } \\
\text { transporte de } \\
\text { pasajero }\end{array}$ \\
\hline $\begin{array}{l}\text { ISR por la } \\
\text { conservación } \\
\text { SAS }\end{array}$ & - & 5 años & $\begin{array}{l}\text { Nacional, } \\
\text { privada }\end{array}$ & $\begin{array}{c}\text { Productos De } \\
\text { Colombia (Prodeco) } \\
\text { S.A. Minas } \\
\text { Calenturitas, } \\
\text { Carbones De La } \\
\text { Jagua (CDJ) S.A., } \\
\text { Consorcio Minero } \\
\text { Unido (CMU), } \\
\text { Sociedad Portuaria } \\
\text { Puerto Nuevo S.A., } \\
\text { Unión Temporal Rio } \\
\text { Córdoba, Colombian } \\
\text { Natural Resourse }\end{array}$ & - & $\begin{array}{c}\text { Proyectos de } \\
\text { rescate y } \\
\text { Reubicación de } \\
\text { fauna silvestre y } \\
\text { Consultorías } \\
\text { Biológico } \\
\text { Ambientales para el } \\
\text { control y manejo } \\
\text { adecuado de fauna } \\
\text { silvestre }\end{array}$ \\
\hline
\end{tabular}




\begin{tabular}{|c|c|c|c|c|c|c|}
\hline \multicolumn{7}{|c|}{ Tabla 1 (continuación) } \\
\hline Servividrios & - & 42 años & $\begin{array}{l}\text { Privada, } \\
\text { nacional }\end{array}$ & $\begin{array}{c}\text { Prodeco. } \\
\text { No disponible } \\
\text { información del resto } \\
\text { de clientes }\end{array}$ & - & $\begin{array}{l}\text { Proveedor de } \\
\text { cristales y espejos }\end{array}$ \\
\hline $\begin{array}{l}\text { Interglobal } \\
\text { LTDA }\end{array}$ & 3,256 & 20 años & $\begin{array}{l}\text { Nacional, } \\
\text { privada }\end{array}$ & $\begin{array}{c}\text { CNR, DOLE, DPA, } \\
\text { Drummond Limited, } \\
\text { Ecopetrol, } \\
\text { Euromarmol, Fenoco, } \\
\text { Gecolsa, Gracetales, } \\
\text { Granabastos, Grupo } \\
\text { Coremar, Panorama, } \\
\text { Pricesmart, Procaps, } \\
\text { Prodeco, Proficol, } \\
\text { Promigas, RCN } \\
\text { Radio, Retycol, Salud } \\
\text { Total, UCPL. }\end{array}$ & $\begin{array}{c}\$ 31,395,625 \\
\text { Activos totales. } \\
2016 \text { (Unidades } \\
\text { en Miles) }\end{array}$ & $\begin{array}{l}\text { Servicios de } \\
\text { seguridad y } \\
\text { vigilancia }\end{array}$ \\
\hline $\begin{array}{l}\text { Xylem Water } \\
\text { Solutions } \\
\text { Colombia S } \\
\text { A S }\end{array}$ & 82 & 12 años & $\begin{array}{l}\text { Internacion } \\
\text { al, privada }\end{array}$ & $\begin{array}{c}\text { Productos vendidos } \\
\text { en más de } 150 \text { países } \\
\text { a través de una fuerza } \\
\text { de ventas líder en el } \\
\text { mercado }\end{array}$ & $\begin{array}{c}\$ 28,080,890 \\
\text { Activos totales. } \\
2016 \text { (Unidades } \\
\text { en Miles) }\end{array}$ & $\begin{array}{c}\text { Abastecimiento, } \\
\text { transporte y } \\
\text { tratamiento del agua }\end{array}$ \\
\hline $\begin{array}{l}\text { Compass } \\
\text { Group } \\
\text { Colombia S } \\
\text { A S }\end{array}$ & 6,706 & $\begin{array}{l}77 \text { años } \\
11 \text { años } \\
\text { en } \\
\text { Colombia }\end{array}$ & $\begin{array}{l}\text { Internacion } \\
\text { al, privada }\end{array}$ & $\begin{array}{c}\text { Andercol, } \\
\text { Cerro Matoso S.A., } \\
\text { Chevron Petroleum } \\
\text { Company, } \\
\text { Clínica del Country, } \\
\text { Clínica de Occidente, } \\
\text { Colpapel } \\
\text { Coordinadora, } \\
\text { Drummond Ltd., } \\
\text { Ecopetrol, } \\
\text { Continental Gold, } \\
\text { Pintuco, Prodeco }\end{array}$ & $\begin{array}{c}\$ 123,697,351 \\
\text { Activos totales. } \\
2016 \text { (Unidades } \\
\text { en Miles) }\end{array}$ & $\begin{array}{c}\text { Servicios de } \\
\text { Alimentación y de } \\
\text { soporte }\end{array}$ \\
\hline $\begin{array}{l}\text { Garzón y } \\
\text { Salazar } \\
\text { S.A.S }\end{array}$ & 92 & 33 años & $\begin{array}{l}\text { Nacional, } \\
\text { privada }\end{array}$ & $\begin{array}{c}\text { Prodeco } \\
\text { No disponible } \\
\text { información del resto } \\
\text { de clientes }\end{array}$ & $\begin{array}{c}\$ 5,566,755 \\
\text { Activos totales. } \\
2016 \text { (Unidades } \\
\text { en Miles) }\end{array}$ & $\begin{array}{l}\text { Suministro de } \\
\text { recursos humanos }\end{array}$ \\
\hline $\begin{array}{l}\text { Manpower } \\
\text { professional } \\
\text { LTDA }\end{array}$ & 4,263 & $\begin{array}{l}70 \text { años } \\
26 \text { años } \\
\text { en } \\
\text { Colombia }\end{array}$ & $\begin{array}{l}\text { Internacion } \\
\text { al, privada }\end{array}$ & $\begin{array}{l}\text { Aproximadamente } \\
400.000 \text { clientes }\end{array}$ & $\begin{array}{c}\$ 46,538,880 \\
\text { Activos totales. } \\
2016 \text { (Unidades } \\
\text { en Miles) }\end{array}$ & $\begin{array}{l}\text { Líder mundial en la } \\
\text { industria de los } \\
\text { servicios de empleo }\end{array}$ \\
\hline
\end{tabular}

De acuerdo con la Tabla 1, las empresas objeto de estudio corresponden a las empresas contratistas de la mina Calenturitas, empresa multinacional dedicada a la extracción de carbón en el departamento de Cesar, Colombia. La tabla 1 presenta las principales características de las contratistas analizadas. La información de la tabla 1 fue obtenida de la página web de estas empresas y de portales de información como: einforma, EMIS y empresas.protafolio. Adicionalmente, se destaca que, en su mayoría, los informantes claves de la presente investigación corresponden a supervisores de operaciones o coordinadores de las empresas.

Las contratistas incluidas en la tabla 1 se encuentran agrupadas de acuerdo con la actividad que realiza en la empresa minera objeto de estudio. En este sentido, se identifica un primer grupo de empresas que se orienta a la comercialización y reparación de equipos mineros, posteriormente, se identifica un segundo grupo de empresas que apoyan actividades de construcción, tales como carreteras y vías de ferrocarril. El tercer grupo de empresas tiene como actividad las perforaciones geológicas requeridas para la operación minera. Seguidamente, se identifican empresas orientadas al control de calidad de combustibles sólidos, el movimiento de carga, la realización de procesos logísticos y de transporte. Las contratistas anteriormente descritas, apoyan los procesos centrales o misionales de la empresa minera analizada. De otro lado, se identifica un conjunto de contratistas que soportan los procesos de apoyo y estratégicos de la empresa, mediante servicios como: suministro de talento humano, seguridad, vigilancia y alimentación para el personal de la empresa minera.

Adicionalmente, el artículo utiliza la técnica de la encuesta, mediante un cuestionario de preguntas cerradas, tomado del artículo científico titulado, "Innovation or imitation? The role of organizational culture" de los autores Naranjo et al. (2011) considerando que corresponde a un instrumento validado para la medición de los rasgos y tipos de cultura innovadora. El cuestionario mide cuatro rasgos de la cultura innovadora: características de la organización dominante, gestión del personal, vínculo organizacional y criterios de 
éxito. Cada uno de los rasgos tiene cuatro opciones de respuesta (Tal como se presenta en las figuras 1, 2, $3,4)$. Estas opciones corresponden a las definiciones de los cuatro tipos de cultura del modelo de Cameron y Quinn (1999): de clan, adhocrática, de mercado y jerárquica. A los encuestados se les pidió asignar 100 puntos entre los cuatro ítems de cada sección, dependiendo de cuán similares eran las descripciones para su organización.

\section{RESULTADOS}

A continuación se presentan los resultados de cada uno de los rasgos de la cultura innovadora en las empresas analizadas.

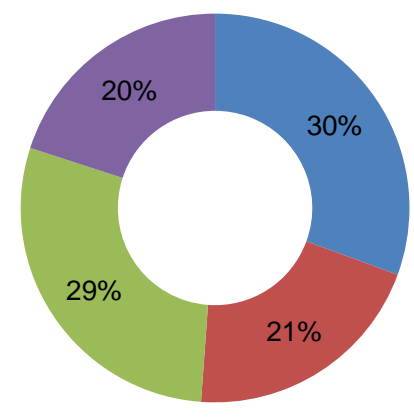

- Opción 1. (Tipo de cultura de clan)

- Opción 2. (Tipo de cultura adhocrática)

- Opción 3. (Tipo de cultura de mercado)

- Opción 4. (Tipo de cultura jerárquica)

Fig. 1: Rasgo de la cultura innovadora: Características dominantes de la organización

La figura 1 presenta la distribución de los porcentajes correspondientes al rasgo de la cultura innovadora: Características dominantes de la organización. En primer lugar, el mayor porcentaje corresponde a la opción 1, relacionada con la afirmación de que la empresa es como una familia extensa, en la cual los trabajadores comparten mucho entre ellos. En segundo lugar, se ubica la opción 3, que hace referencia al planteamiento de que la empresa está orientada a los resultados, dado que existe una preocupación importante con el trabajo y los trabajadores son muy competitivos y orientados a los logros. En tercer lugar, se sitúa la opción 2, la cual afirma que la empresa es un lugar dinámico y emprendedor, en el que las personas están dispuestas a unir esfuerzos y tomar riesgos. En cuarto lugar, se registra la opción 4, asociada al planteamiento de que la empresa es un lugar controlado y estructurado, en el que los procedimientos formales generalmente rigen las actividades que realizan las personas.

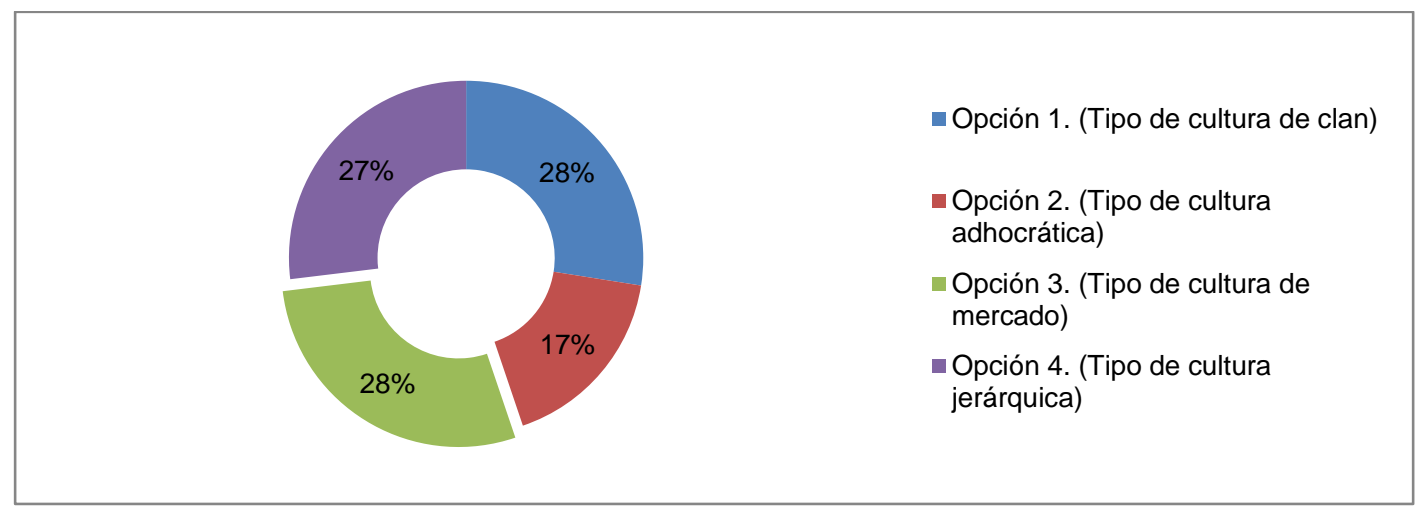

Fig. 2: Rasgo de la cultura innovadora: Gestión del personal

La figura 2 presenta la distribución de porcentajes correspondiente al rasgo de la cultura innovadora: Gestión del personal. En primer lugar, el mayor porcentaje corresponde a la opción 3 que establece que la empresa se caracteriza por la competitividad, las altas exigencias y los logros. En segundo lugar, se ubica la opción 1 correspondiente a la afirmación de que la empresa se caracteriza por el trabajo en equipo, el consenso y la participación. En tercer lugar, se ubica la opción 4, asociada al planteamiento de que la empresa se caracteriza por la seguridad del empleo, la conformidad, la previsibilidad y la estabilidad en las relaciones. En cuarto lugar, la opción 2 registra el menor porcentaje. Esta última opción se relaciona con el hecho de que la empresa se caracteriza por tomar riesgos individuales, la innovación, la libertad y el carácter único. 


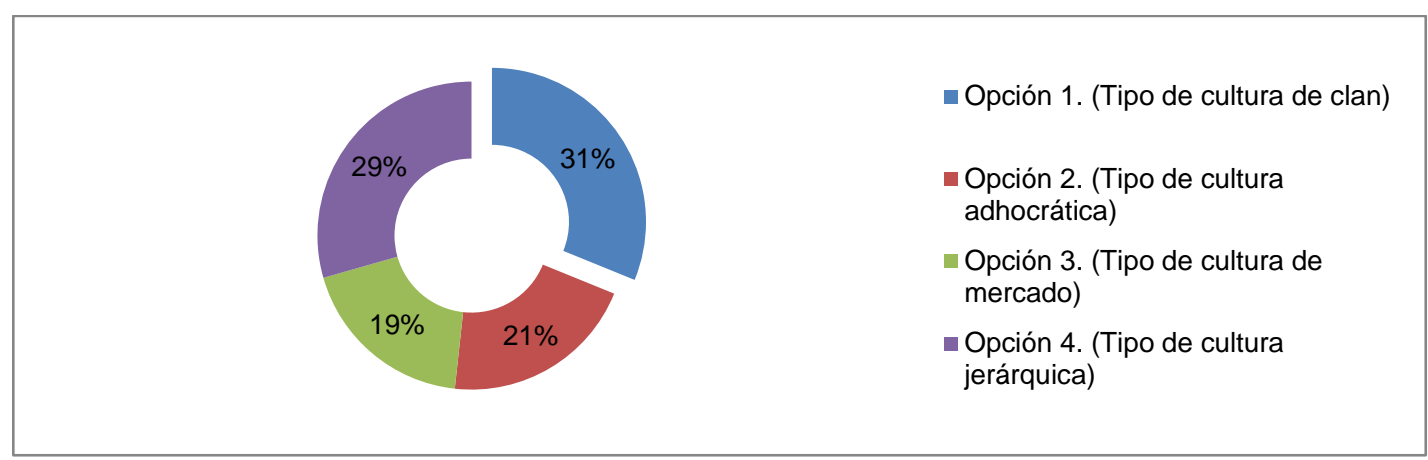

Fig. 3: Rasgo de la cultura innovadora: Vinculo organizacional

La figura 3 presenta la distribución de porcentajes correspondiente al rasgo de la cultura innovadora: Vinculo organizacional. En primer lugar, el mayor porcentaje corresponde a la opción 1 que hace referencia a que la empresa se caracteriza por la lealtad, compromiso organizacional, confianza mutua y trabajo en equipo. En segundo lugar, se ubica la opción 4, asociada con el planteamiento de que la empresa se caracteriza por la existencia de reglas formales y políticas de mantenimiento y la importancia de la jerarquía. En tercer lugar, se establece la opción 2, correspondiente al planteamiento de que la empresa se caracteriza por el compromiso con la innovación y hace énfasis en estar a la vanguardia. En cuarto lugar, la opción 3 obtiene el menor porcentaje; esta opción plantea que la empresa se caracteriza por la agresividad, el liderazgo en el mercado y el logro de los objetivos.

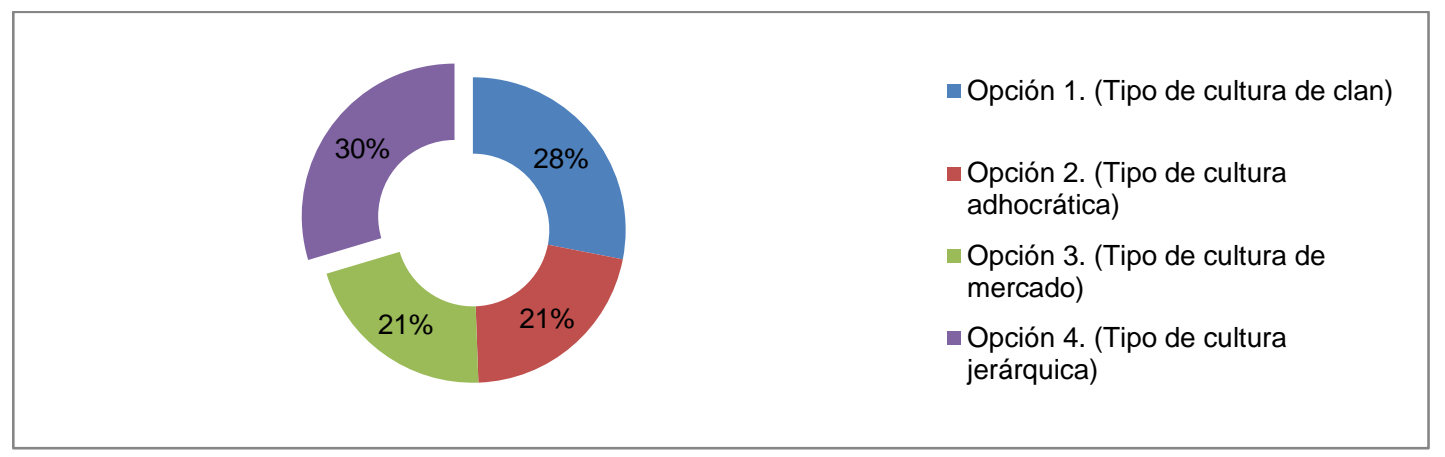

Fig. 4: Rasgo de la cultura innovadora: Criterios de éxito

La figura 4 presenta la distribución de porcentajes correspondiente al rasgo de la cultura innovadora: Criterios de éxito. En primer lugar, el mayor porcentaje corresponde a la opción 4 que plantea que para la empresa son críticos los procesos de entrega eficiente y confiable, la programación detalla y la producción de bajo costo. En segundo lugar, se ubica la opción 1, asociada al planteamiento de que la empresa se caracteriza por el desarrollo de los recursos humanos, el trabajo en equipo, el compromiso de los empleados y la preocupación por las personas. En tercer lugar, se registra la opción 2, asociada al planteamiento de que la empresa se caracteriza por tener los productos únicos o más nuevos, es decir, es líder de producto e innovadora. En cuarto lugar, el menor porcentaje corresponde a la opción 3, que plantea que para la empresa es clave liderar y superar el liderazgo competitivo del mercado.

Para efectos de identificar el tipo de cultura organizacional presente en las empresas contratistas de las minas de explotación de carbón del departamento del Cesar, se tabularon los porcentajes obtenidos de la aplicación de las encuestas. Posteriormente, se establecieron los promedios de cada uno de los ítems que se asocian a cada uno de los tipos de cultura organizacional analizados en el modelo de Cameron y Quinn (1999) retomado por Naranjo et al. (2012). En este sentido, se obtiene la tabla con los siguientes promedios para establecer el nivel de presencia de cada uno de los cuatro tipos de cultura analizados (Tabla 2):

Tabla 2: Estimación del porcentaje de presencia de las culturas en las empresas

\begin{tabular}{|l|c|c|c|c|c|c|}
\hline $\begin{array}{l}\text { Característical } \\
\text { Tipo de cultura }\end{array}$ & $\begin{array}{c}\text { Características } \\
\text { dominantes (\%) }\end{array}$ & $\begin{array}{c}\text { Gestión del } \\
\text { personal (\%) }\end{array}$ & $\begin{array}{c}\text { Vinculo } \\
\text { organizacional } \\
(\%)\end{array}$ & $\begin{array}{c}\text { Criterio de } \\
\text { éxito (\%) }\end{array}$ & $\begin{array}{c}\text { Promedio } \\
\text { (\%) }\end{array}$ & $\begin{array}{c}\text { Orden de presencia } \\
\text { en las empresas (1 } \\
\text { mayor presencia) }\end{array}$ \\
\hline 1. Clan & 31 & 28 & 31 & 28 & 30 & 1 \\
\hline 2. Adhocrática & 21 & 17 & 21 & 21 & 20 & 4 \\
\hline 3. Mercado & 29 & 28 & 19 & 21 & 24 & 3 \\
\hline 4. Jerárquica & 20 & 27 & 29 & 30 & 27 & 2 \\
\hline
\end{tabular}


De acuerdo con la Tabla 2, la cultura con mayor presencia en las empresas analizadas en la de clan, con un $30 \%$ de presencia; seguidamente, la cultura jerárquica, con un $27 \%$; en tercera medida, la cultura de mercado con un $24 \%$ de presencia y, por último, la cultura adhocrática con un $20 \%$, es la tipología con menor presencia en las empresas contratistas analizadas. En contraste con literatura, la tipología de cultura organizativa del modelo de Cameron y Quinn (1999) que más favorece la innovación es el adhocrática (Naranjo et al., 2012), dado que los dos valores principales que la caracterizan son la flexibilidad y la orientación externa. Además, los rasgos que Cameron y Quinn (1999) y Naranjo et al. (2012) utilizan para definir este tipo de cultura (características dominantes, gestión de personal, vínculo organizacional y criterio de éxito) incluyen valores asociados a la flexibilidad, como la iniciativa, la autonomía y la asunción de riesgos.

Con el fin de profundizar en el resultado anterior, se realiza un análisis ANOVA con el fin de determinar si existen diferencias significativas entre los tipos de cultura analizados, para tal fin se promedian cada uno de los puntajes objetivos en el tipo de cultura, obtenido los resultados presentados en la tabla 3.

Tabla 3: ANOVA

\begin{tabular}{|l|r|r|r|r|r|}
\hline & Suma de cuadrados & gl & Media cuadrática & F & \multicolumn{1}{c|}{ Sig. } \\
\hline Entre grupos & 181,50 & 3 & 60,50 & 4,25 &, 029 \\
Dentro de grupos & 170,50 & 12 & 14,20 & & \\
Total & 352,00 & 15 & & & \\
\hline
\end{tabular}

Debido a que el nivel de significancia es menor que 0.05 , lo que indica que existen diferencias significativas entre los grupos analizados, en este caso, entre los tipos de cultura innovadora en las empresas contratistas. Para establecer las culturas que presentan estas diferencias, se realiza una prueba post hoc, en este caso el HSD Tukey, obteniendo los resultados presentados en la tabla 4.

Tabla 4: Comparaciones múltiples ( ${ }^{*}$. La diferencia de medias es significativa en el nivel 0.05.)

\begin{tabular}{|lc|c|c|c|c|c|}
\hline \multirow{2}{*}{ (I) Tipo_de_cultura } & (J) Tipo_de_cultura & Diferencia de & \multirow{2}{*}{ medias (I-J) } & Error estándar & \multirow{2}{*}{ Sig. } & \multicolumn{2}{|c|}{ Intervalo de confianza al 95\% } \\
\hline \multirow{4}{*}{ De clan } & Adhocrática & $9,50^{*}$ & 2,66 &, 01 & 1,58 & 17,41 \\
& Mercado & 5,25 & 2,66 &, 25 & $-2,66$ & 13,16 \\
& Jerárquica & 5,25 & 2,66 &, 25 & $-2,66$ & 13,16 \\
\hline \multirow{4}{*}{ Adhocrática } & De clan & $-9,50^{*}$ & 2,66 &, 01 & $-17,41$ & $-1,58$ \\
& Mercado & $-4,25$ & 2,66 &, 41 & $-12,16$ & 3,66 \\
& Jerárquica & $-4,25$ & 2,66 &, 41 & $-12,16$ & 3,66 \\
\hline \multirow{4}{*}{ Mercado } & De clan & $-5,25$ & 2,66 &, 25 & $-13,16$ & 2,66 \\
& Adhocrática & 4,25 & 2,66 &, 41 & $-3,66$ & 12,16 \\
& Jerárquica &, 00 & 2,66 & 1,00 & $-7,91$ & 7,91 \\
\hline \multirow{3}{*}{ Jerárquica } & De clan & $-5,25$ & 2,66 &, 25 & $-13,16$ & 2,66 \\
& Adhocrática & 4,25 & 2,66 &, 41 & $-3,66$ & 12,16 \\
& Mercado &, 00 & 2,66 & 1,00 & $-7,91$ & 7,91 \\
\hline
\end{tabular}

A partir de las comparaciones múltiples presentadas en la Tabla 4, solo se presentan diferencias significativas (Sig. < 0.05) entre la cultura de clan (mayor presencia) y la cultura adhocrática (menor presencia). Este resultado contrasta con la literatura que indica que, la cultura adhocrática es la que mayor favorece la innovación (Naranjo et al., 2012), debido a su orientación a flexibilidad y al exterior, y a los valores característicos como la creatividad, la libertad/autonomía, la tolerancia al riesgo, la suficiencia de recursos, la orientación estratégica hacia el cliente y la orientación hacia el aprendizaje continuo. Por lo anterior, resulta importante aplicar estrategias para que las empresas analizadas logren una cultura orientada a la innovación, que les permita crecer en el mercado y satisfacer las expectativas de sus grupos de interés. 
Adicionalmente, de acuerdo con Naranjo et al. (2012) cada uno de los rasgos que se utilizan para definir los tipos de cultura (características dominantes, gestión de personal, vínculo organizacional y criterio de éxito) de forma independiente, favorecen la innovación, no obstante, el mayor efecto se produce cuando todos los rasgos de la cultura son coherentes entre sí. Así, dado que el tipo de cultura adhocrática es la que tiene menor presencia es fundamental implementar acciones al interior de los equipos de trabajo para aumentar el compromiso, el sentido de pertenencia y la generación de ideas. Al respecto, es necesario crear y definir estructuras y procesos para la generación de ideas provenientes de todos los grupos de interés, acompañado de una gestión eficiente y sistemática de proyectos (Rech et al., 2015).

\section{CONCLUSIONES}

Al analizar los rasgos que definen la cultura organizacional de las empresas contratistas de las minas de explotación de carbón del departamento del cesar, se concluye lo siguiente: En primer lugar, en referencia a las características de la organización dominante, el rasgo predominante, es la concepción de la empresa como una familia extensa, en la que los trabajadores comparten mucho entre ellos. Este elemento se asocia a un tipo de cultura de clan. En segundo lugar, frente a la gestión de personal, los rasgos predominantes son, el fomento del trabajo en equipo, el consenso y la participación a la empresa. Este hecho se asocia a la cultura de clan. Paralelamente, las empresas se conciben como espacios de competitividad, altas exigencias y logros, como un aspecto característico de la cultura de mercado. En tercer lugar, el rasgo predominante en referencia al vínculo organizacional, corresponde a la concepción de la empresa como un espacio caracterizado por la lealtad, el compromiso organizacional, la confianza mutua y el trabajo en equipo, elementos propios de las culturas de clan. Similarmente, en el rasgo de criterios de éxito, se destacan los rasgos asociados a una cultura de clan, debido a la promoción del desarrollo de los recursos humanos, el compromiso de los empleados y la preocupación por las personas en la mayor parte de las empresas analizadas. En consecuencia, el tipo de cultura predominante en las empresas analizadas es la cultura de clan, seguido de la jerárquica, la cultura de mercado y la adhocrática, respectivamente.

\section{REFERENCIAS}

Arancibia Carvajal, S., M. Donoso Pérez, R. Venegas Cabello y C. Cárdenas Espinosa, Identificación de Factores Clave en la Cultura de Innovación, El Caso de la Mediana Minería en Chile, Journal of Technology Management and Innovation, 10(01), 132 - 145 (2015)

Ayala, P., Cultura Organizacional e Innovación, Gestión de Personas y Tecnología, 19, 27 - 33 (2014)

Bravo Ibarra, E.R. y L. Herrera, Capacidad De Innovación y Configuración de Recursos Organizativos, Intangible Capital, 5(3), 301-320 (2009)

Calderón Hernández, G. y J. Naranjo Valencia, Perfil Cultural de las Empresas Innovadoras. Un Estudio de Caso en Empresas Metalmecánicas, Cuadernos de Administración, 20(34), 161-189 (2007)

Cameron, K. y R. Quinn, Diagnosing and Changing Organizational Culture, The Jossey-Bass Business and Management Series, San Francisco, CA (1999)

Departamento Administrativo Nacional de Estadísticas, Encuesta de Desarrollo e Innovación Tecnológica EDIT, Bogotá D.C., Colombia (2015)

Departamento Administrativo Nacional de Estadísticas DANE, Encuesta de Desarrollo e Innovación Tecnológica Sector Servicios, Comunicado de prensa, Bogotá D.C., Colombia (2014)

Fernández Jardón, C.M., Determinantes de la Capacidad de Innovación en Pymes Regionales, Revista de Administração da Universidade Federal de Santa María, 5, 749-766 (2012)

González, J. M., Innovar: un imperativo para competir en la economía mundial. Revista de Ingeniería, U. d. Andes, 38, $52-59$ (2013)

Guzmán, J. A., La Innovación Empresarial y La Cultura Organizacional. 3c Empresa, 4(3), (2015)

Hernandez, S.,R., C. Fernández y P. Baptista, Metodología de la investigación; 4ª Ed., Mc Graw Hill, México. D.F, Mexico (2014)

López González, C. y J. Robledo Velásquez, Una aproximación a la gestión de capacidades de innovación en la pyme colombiana, Gestión \& Sociedad, 7(2), 11-30 (2014)

Mohr, J.J., S. Sengupta y S. Slater, Marketing of High-Technology Products and Innovations; $3^{\text {rd }}$ Edition, Pearson (2010)

Naranjo Valencia, J., D. Jiménez Jiménez y R. Sanz Valle, Innovation or Imitation? The Role of Organizational Culture, Management Decision, 49(1), 55 - 72 (2011)

Naranjo Valencia, J., D. Jiménez y R. Sanz Valle, ¿Es La Cultura Organizativa un Determinante de la Innovación en la Empresa?; Cuadernos de Economía y Dirección de la Empresa, 15(02), 63 - 72 (2012) 
Naranjo Valencia, J.C. y G. Calderón Hernández, Construyendo Una Cultura de Innovación. Una Propuesta de Transformación Cultural, Estudios Gerenciales, 31(135), 223-236 (2015)

OECD y Eurostat, Manual de Oslo, Guía Para la Recogida e Interpretación de Datos Sobre Innovación, Organizacion Para la Cooperación y El Desarrollo Económico y Oficina de Estadísticas de las Comunidades Europeas, European Communities: Grupo Tragsa (2005)

Pertuz Peralta, V. y A. Pérez, Modelo De La Cultura Organizacional Innovadora, En Caficultores Del Departamento Del Cesar - Colombia, Revista investigación desarrollo innovación, 6(2), 117 - 130 (2016)

Rech, I., J. Rudnik, C. M. Pereira, M. A. Duarte y A. Schlemmer, Análise da Cultura Inovadora em uma Indústria de Colchões, Revista Espacios, 36(14), 8 - 24 (2015)

RICYT, OAS, CYTED, COLCIENCIAS y OCYT, Normalización de Indicadores de Innovación Tecnológica En América Latina y El Caribe: Manual De Bogotá, Bogotá D.C., Colombia (2001)

Rosenbusch, N., J. Brinckmann y A. Bausch, Is Innovation Always Beneficial? A Meta-analysis of the relationship between innovation and performance in SMES, Journal of Business Venturing, 26(4), $441-457$ (2011)

Rueda Barrios, G. y A. Rodenes, Hacia Una Cultura Innovadora En Las Empresas, Departamento de Organización de Empresas de la Universidad Politécnica de Valencia, España (2010)

Ruiz, Y. B. y J. C. Naranjo, La Investigación Sobre Cultura Organizacional En Colombia: Una Mirada Desde La Difusión En Revistas Científicas, Diversitas, Perspectivas en Psicología, 8(2), 285-307 (2012)

Sanabria, N., C. Acosta Prado y G. Rodríguez Albor, Condiciones Para La Innovación, Cultura Organizacional Y Sostenibilidad De Las Organizaciones, Semestre Económico, 18(37), 157 - 176 (2015)

Saunila, M. y J. Ukko, Intangible aspects of innovation capability in SMEs: Impacts of size and industry; Journal of Engineering and Technology Management, 33, 32-46 (2014)

Saunila, M., S. Pekkola y J. Ukko, The relationship between innovation capability and performance: the moderating effect of measurement, International Journal of Productivity and Performance Management, 63(2), 234-249 (2014)

Schumpeter, J., The Theory of Economic Development; Harvard University Press, Cambridge (1934)

Siguaw, J., P. Simpson y C. Enz, Conceptualizing innovation orientation: a framework for study and integration of innovation research, Journal of Product Innovation Management, 23(6), 556 - 574 (2006)

Souto, J., Gestión de una cultura de innovación basada en las personas; Journal of Technology Management and Innovation, 10(3), 60 - 65 (2015)

Tejeiro Koller, M., Medición de la cultura de innovación: depuración con cuatro estudios; Intangible Capital, 10(3), 467504 (2014) 